\title{
QUASI-PERIODIC (QP) EMISSIONS AS OBSERVED BY JUNO WAVES
}

\author{
G. B. Hospodarsky*, M. Imai*, W. S. Kurth*, D. A. Gurnett*, \\ and S. J. Bolton ${ }^{\dagger}$
}

\begin{abstract}
A number of quasi-periodic (QP) signatures with periods of $<1$ minute to tens of minutes have been detected in the wave and particle observations of the Jovian magnetosphere by a number of different spacecraft. QP variation with similar periods has also been reported of the brightness of the Jovian aurora. In radio and plasma wave observations by Voyager, these QP emissions were given the name Jovian type III radio bursts due to their dispersion spectral shape similar to solar type III radio bursts, though on much shorter time scales. Ulysses observations found that these emissions were made up primarily of two periods, 15 and 40 minutes, and renamed the emissions quasi-periodic emissions because the Jovian type III designation might imply a specific generation mechanism. Galileo observations found QP enhancements in the Jovian trapped continuum, but with a much less organized (more random) periodicity. Joint Ulysses, Galileo and Cassini observations showed that many of the events were seen simultaneously by multiple spacecraft at very different locations, suggesting that the emission is beamed in a strobe light or flash bulb like manner. The Juno spacecraft with its low altitude polar orbit around Jupiter provides a new opportunity to examine these QP signatures. The Juno spacecraft Waves instrument detected QP emissions during the approach to Jupiter, prior to the successful orbit insertion on July 5, 2016, and during the initial perijove orbits, including QP emissions detected in the high latitude polar region at very low altitudes. The properties of these emissions as detected by Juno will be discussed.
\end{abstract}

\footnotetext{
* Department of Physics and Astronomy, University of Iowa, Iowa City, IA, USA

$\dagger$ Southwest Research Institute, San Antonio, TX, USA
} 
